\title{
Poster Abstract: Occupancy-based heating control for residential buildings using environmental sensors
}

\author{
Dominic Wörner \\ Dep. of Management, Technology, \\ and Economics \\ ETH Zurich \\ dwoerner@ethz.ch
}

\author{
Thomas von Bomhard \\ Institute of Technology \\ Management \\ University of St. Gallen \\ thomas.vonbomhard@unisg.ch felix.wortmann@unisg.ch
}

\begin{abstract}
Large amounts of energy is wasted in residential buildings because the heating runs round-the-clock although residents are out of home for certain times of a day. It is therefore our aim to develop a retrofit solution for dwellings that automatically detects occupancy and controls the heating accordingly. We demonstrate that consumer-oriented indoorenvironmental sensors can be leveraged to infer occupancy and describe a occupancy-based heating control system on a per-room level. Furthermore, we sketch two forthcoming studies to evaluate our system in a real-world apartment.
\end{abstract}

\section{Introduction}

Heating and cooling of buildings accounts for a major part of global energy consumption, e.g. in 2010, approximately $35 \%$ of the final energy in Germany was used for hot water and space heating. Even more interestingly, more than $70 \%$ of this energy was used in residential buildings [1]. While energy could be saved by decreasing the temperature set point this may also affect the comfort of the occupants. On the other hand the set point could be lowered while the residents are away without affecting the comfort. The problem hereby is that most residents struggle to program their heating schedule [2]. Occupancy detection for residential buildings has been shown to have large impact on space heating and cooling costs. Gupta et al. [3] conducted an experiment with gps-enabled thermostats and showed potential savings of $7 \%$. However, this approach raises privacy concerns and requires that all residents are smartphone users which is unlikely especially in case of families. Gao and Whitehouse [4] developed self-programming thermostats using occupancy data from simple security sensors (passive infrared motion sensors, door reed contacts) and saved up to $15 \%$ in cooling and heating consumption. Although such motion sensors are widely used for occupancy detection, they have strong

Permission to make digital or hard copies of part or all of this work for personal or classroom use is granted without fee provided that copies are not made or distributed for profit or commercial advantage and that copies bear this notice and the full citation on the first page. Copyrights for third-party components of this work must be honored. For all other uses, contact the Owner/Author.

Copyright is held by the owner/author(s).

Buildsys'13, Nov 14 - November 15 2013, Roma, Italy ACM 978-1-4503-2431-1/13/11.

http://dx.doi.org/10.1145/2528282.2528313 limitations when people do not move. In 2009, Lam et al. [5] investigated the use of an ambient sensing infrastructure consisting of $\mathrm{CO}_{2}, \mathrm{CO}$, TVOC, temperature, relative humidity, lightning, motion and noise in office spaces to infer the number of occupants. They achieved an accuracy of $80 \%$ using a Hidden Markov Model and it turned out that the best predictors are $\mathrm{CO}_{2}$ and noise.

Together with the spread of connected consumer products like Netatmo ${ }^{1}$ and Cube Sensors ${ }^{2}$ which provide $\mathrm{CO}_{2}$ and noise sensors besides a variety of other environmental sensors we consider it fruitful to investigate their potential for occupancy-based heating control in dwellings.

\section{System Overview \\ Sensors}

We utilize the Netatmo weather station which provides measurements of $\mathrm{CO}_{2}$, temperature, relative humidity, barometric pressure and noise at a temporal resolution of $5 \mathrm{~min}$. The measurement data is uploaded to the Netatmo server infrastructure and is immediately accessible through a RESTful API.

\section{Occupancy Detection}

Given the collected sensor data our aim is to infer an occupancy profile. In a first step, we use simple heuristics analogous to [5]. Figure 1 shows typical $\mathrm{CO}_{2}$ and noise measurements over the course of a day. Although we plan to detect occupancy in dwellings, the present data is collected in one of our two person offices. The black area denotes occupancy as inferred from the $\mathrm{CO}_{2}$ measurements, the red area from the noise measurements. We see a convincing overlap between the two occupancy estimates and note the arrival of an occupant in the morning, a lunch break between 11:30 and 12:30, and the departure around 18:00. The heuristic for occupancy in the case of $\mathrm{CO}_{2}$ was a increase of $50 \mathrm{ppm}$ between two subsequent measurements. The departure of occupants was detected by a decrease of 50 to $100 \mathrm{ppm}$ between two subsequent measurements. A decrease of more than $100 \mathrm{ppm}$ was seen to be only possible through airing of the office. For a heating control scenario, we can use additionally temperature measurements to distinguish between departure and airing. In the case of noise a threshold value of $37.5 \mathrm{db}$ was used to decide for occupancy. Here, we neglected threshold crossings lasting only one time slot.

\footnotetext{
${ }^{1}$ http://www.netatmo.com

${ }^{2}$ http://cubesensors.com
} 


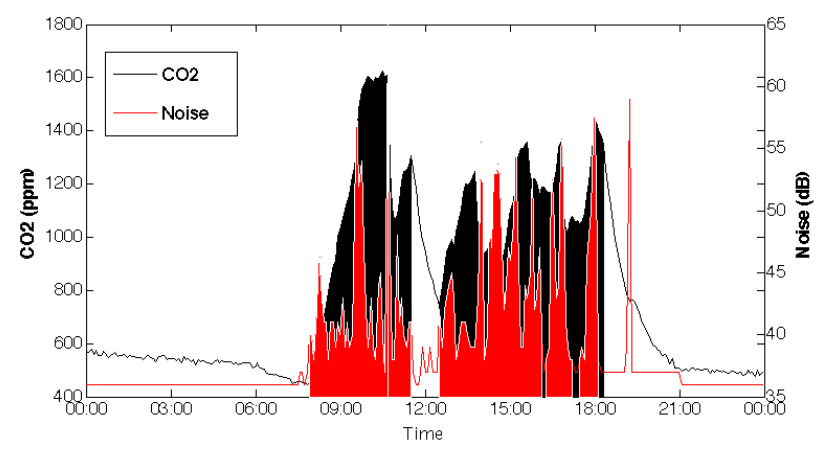

Figure 1. $\mathrm{CO}_{2}$ and noise profile of a workday at the office. The filled areas mark occupancy as given by our heuristic.

\section{Setback Schedule}

The occupancy profile is used to build a setback schedule in order to leverage times of absence to lower the set point temperature. This can be done e.g. with the optimization algorithms described by Gao and Whitehouse [4].

\section{Heating Control}

The typical heating system in Germany, Switzerland and Austria consists of a gas-fired boiler in the basement which supplies hot water to radiators in the individual rooms. In order to control the room temperature individually thermostatic valves are used which are able to restrain the flow through the radiators. We use motorized valves where the set point temperature can be adjusted wirelessly. The communication with the thermostatic valve is accomplished with the Busware $\mathrm{COC}^{3}$ shield mounted on a Raspberry Pi.

\section{Energy Consumption}

In order to infer the energy consumption we can track the gas meter either by a camera or in many cases by a reed switch which produces a signal whenever the magnet attached to the rotating disc inside the meter passes. This approach, while straightforward and cheap, has the disadvantage that gas is also used to heat up water for showering etc. However, the usage of hot water is independent of our intervention and can be eliminated.

\section{Forthcoming Studies}

Occupancy detection using $\mathrm{CO}_{2}$ and noise in an apartment

Since our actual focus is on dwellings we will deploy Netatmo base stations in all major rooms of an apartment with two residents. The residents are both $\mathrm{PhD}$ students which means that the apartment will be empty for a significant time during the workweek. In order to evaluate the validity of the occupancy estimation using $\mathrm{CO}_{2}$ and noise measurements we will deploy two cameras in the hallway such that all doors can be observed as can be seen in Fig. 2. Hence, we will be able to manually assess the occupancy of the individual rooms providing the necessary ground truth. Because of the intrusiveness of the video cameras the experiment will be fixed to a period of 2 weeks. We expect that the collected data will be sufficient to calibrate our occupancy criteria.

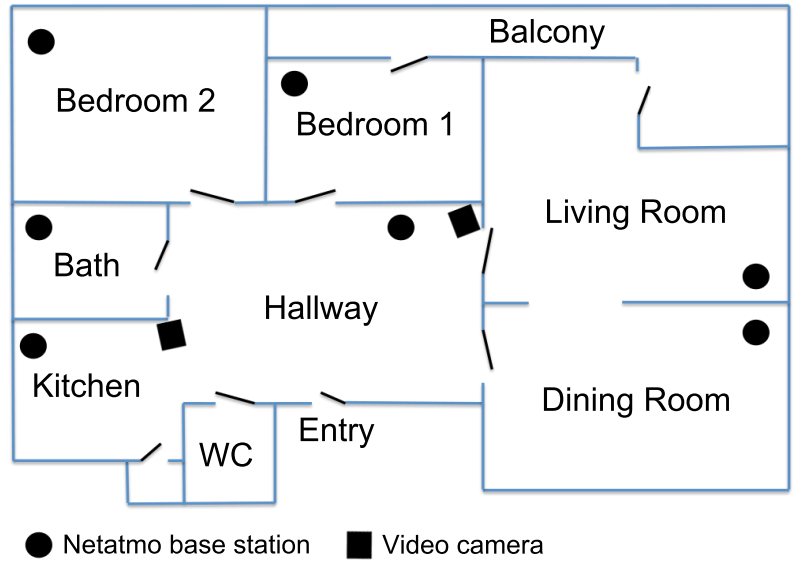

Figure 2. Apartment with locations of Netatmo base stations and cameras.

\section{Per-room heating control using occupancy data}

During the next heating period the collected per-room occupancy profile will be used to build a per-room heating schedule and to control the thermostatic radiator valves accordingly in the apartment shown in Fig. 2. We will record the energy consumption and compare it with the last heating period where our system was not installed after compensating for different outdoor temperatures. The type of residents will likely lead to high savings, since they are both at work for a large fraction of the day. Also, we assume per-room heating in a shared apartment scenario especially reasonable, since there are rooms which are only used by a particular resident.

\section{Conclusions}

We presented a system that infers occupancy from measurements of $\mathrm{CO}_{2}$ and noise by a simple heuristic. We showed that this information can be used to build a setback schedule for thermostatic radiator valves in order to save energy while rooms are not occupied. Furthermore, we sketched two forthcoming studies where we evaluate our approach in a real world apartment.

\section{References}

[1] U. Bigalke, H. Discher, H. Lukas, Y. Zeng, K. Bensmann, and C. Stolte, Der dena Gebäudereport 2012. Deutsche Energie-Agentur GmbH, 2012.

[2] T. Peffer, M. Pritoni, A. Meier, C. Aragon, and D. Perry, "How people use thermostats in homes: A review," Building and Environment, vol. 46, no. 12, pp. 2529-2541, 2011.

[3] M. Gupta, S. S. Intille, and K. Larson, "Adding gps-control to traditional thermostats : An exploration of potential energy savings and design challenges," Pervasive Computing, pp. 1-18, 2009.

[4] G. Gao and K. Whitehouse, "The self-programming thermostat : Optimizing setback schedules based on home occupancy patterns," Proceedings of the First ACM Workshop on Embedded Sensing Systems for Energy Efficiency in Buildings, pp. 67-72, 2009.

[5] K. P. Lam, M. Höynck, B. Dong, B. Andrews, Y. S. Chiou, D. Benitez, and J. Choi, "Occupancy detection through an environmental sensor network in an open-plan office building center for building performance and diagnostics," Proceedings of Building Simulation 09, 2009.

\footnotetext{
${ }^{3}$ http://busware.de/tiki-index.php?page=COC
} 